\title{
Sequential decisions in the college admissions problem
}

\author{
José Alcalde ${ }^{\mathrm{a}}$, Antonio Romero-Medina ${ }^{\mathrm{b}, *}$ \\ ${ }^{a}$ Departamento Fonaments de l'Anàlisi Econòmica, University of Alicante, 03071 Alicante, Spain \\ ${ }^{\mathrm{b}}$ Departamento de Economía, Universidad Carlos III de Madrid, 28903 Getafe, Spain
}

\begin{abstract}
This paper models a contractual process by a sequential mechanism, which mimics matching procedures for many to one real life matching problems. In this framework, we provide a family of mechanisms implementing the student optimal matching in Subgame Perfect Equilibrium (SPE).
\end{abstract}

Keywords: Matching problems; Sequential mechanisms; Stability

JEL classification: $\mathrm{C} 78 ; \mathrm{D} 78$

\section{Introduction}

This paper models contractual processes by sequential mechanisms, which mimic matching procedures for many to one real life matching markets. In this framework, we propose a family of mechanisms implementing the students' optimal allocation in Subgame Perfect Equilibrium (SPE).

The college admissions problem models a contractual process in bilateral markets where monetary transfers are not relevant or can be embodied in agents' preferences. In this paper, we analyze a "hierarchic dictatorship" mechanism inspired by real life allocation processes; in particular, the mechanism used in Spain to allocate civil servants and to distribute residency positions among

* Corresponding author. Tel.: +34 91624 9752; fax: +34 916249875 .

E-mail address: aromero@eco.uc3m.es (A. Romero-Medina). 
physicians. We show how the only matching supported by any Subgame Perfect Nash Equilibrium for this mechanism is the (unique) stable matching that is Pareto optimal from the students' point of view. Moreover, this result is independent from the order in which students are ranked.

Given the symmetry between the two sides of the market, we also investigate the possibility of having a result similar to that of our Theorem 3.1, when the roles played by students and colleges are exchanged. This similarity holds in some matching mechanisms, see Alcalde and Romero-Medina (2000) in the same framework and Alcalde et al. (1998) for job markets. We show that the properties of the mechanism are not satisfied if the proposals are made by the colleges. We point out two features: (i) the matching supported by Subgame Perfect Nash Equilibria depends on the order in which colleges select their strategies and (ii) the equilibrium matching is not necessarily Pareto efficient from the colleges' point of view.

The rest of the paper is organized as follows. Section 2 introduces the basic model. Section 3 presents and analyzes the mechanism, called here the "students-sequentially-propose-and-colleges-choose" mechanism. Conclusions are presented in Section 4.

\section{The model}

We consider a college admissions problem with $n$ colleges and $m$ students. Let $C=\left\{c_{1}, \ldots, c_{n}\right\}$ and $S=\left\{s_{1}, \ldots, s_{m}\right\}$ be the set of colleges and students, respectively. Each college $c_{i}$ has preferences $P\left(c_{i}\right)$ over groups of students and each student $s_{j}$ has preferences $P\left(s_{j}\right)$ over $C \bigcup\left\{s_{j}\right\}$. Preferences are linear orders. A college admissions problem, or simply a problem, is a list $\{C, S ; P\}$, where $P=\left\{P\left(c_{1}\right), \ldots\right.$, $\left.P\left(c_{n}\right), P\left(s_{1}\right), \ldots, P\left(s_{m}\right)\right\}$ is the profile of preferences.

An allocation for such a problem, or matching, is a mapping $\mu$ from $C \cup S$ into $2^{S} \cup C$ satisfying

(i) for all $c_{i} \in C, \mu\left(c_{i}\right) \in 2^{S}$,

(ii) for all $s_{j} \in S, \mu\left(s_{j}\right) \in C \bigcup\left\{s_{j}\right\}$, and

(iii) for each pair $\left(c_{i}, s_{j}\right) \in C \times S,\left[\mu\left(s_{j}\right)=c_{i} \Leftrightarrow s_{j} \in \mu\left(c_{i}\right)\right]$.

From here on, $C$ and $S$ are fixed. Thus we can identify a college admissions problem $\{C, S ; P\}$ with the preference profile $P{ }^{1}$ Let $\mathcal{M}$ be the set of all possible matchings $\mu$. Finally, $\mathbb{P}$ denotes the set of (potential) college admissions problems.

Let $P$ be a college admissions problem. Given a set of students $A \subseteq S$, we denote by $C h_{i}(A)$ the maximal element on $2^{A}$ under the linear order $P\left(c_{i}\right)$.

Definition 2.1. Let $P$ be a college admissions problem. A matching $\mu$ is said to be:

(a) Individually rational for $P$ iff

(i) $C h_{i}\left(\mu\left(c_{i}\right)\right)=\mu\left(c_{i}\right)$ for all $c_{i} \in C$, and

(ii) for all $s_{j} \in S, c_{i} \in C\left[s_{j} P\left(s_{j}\right) c_{i} \Rightarrow s_{j} \notin \mu\left(c_{i}\right)\right]$.

\footnotetext{
${ }^{1}$ For the sake of simplicity, we will employ the same notation for a preference profile and the related college admissions problem. The context will clarify whether $P$ denotes a matching problem or simply a preference profile.
} 
(b) Blocked by a pair $\left(c_{i}, s_{j}\right) \in C \times S$ iff

(i) $c_{i} P\left(s_{j}\right) \mu\left(s_{j}\right)$, and

(ii) $s_{j} \in C h_{i}\left(\mu\left(c_{i}\right) \cup\left\{s_{j}\right\}\right)$. A pair $\left(c_{i}, s_{j}\right)$ which blocks $\mu$ is called a blocking pair for $\mu$.

(c) (Pair-wise) stable if it is individually rational and there is no pair blocking it.

Finally, we assume that colleges' preferences with regard to groups of students are substitutive. That is, for any two students $s_{j} \neq s_{k}$ if $s_{j}$ belongs to $C h_{i}(A)$, then she also belong to $C h_{i}\left(A \backslash\left\{s_{k}\right\}\right)$. This assumption is usual in the literature and guarantees non-emptiness of the set of stable allocations. (See Theorem 6.5 in Roth and Sotomayor, 1990.) Note that, when colleges' preferences are substitutive, the set of (pair-wise) stable allocations coincides with the core of the related college admissions problem. ${ }^{2}$ That is, given a stable allocation, no group of agents can find a matching to improve the welfare of all its members without being matched with agents outside this group. Furthermore, if colleges' preferences satisfy substitutability, the set of stable allocations has a latticial structure. This property guarantees (i) the existence of a single stable allocation which is Pareto optimal from the point of view of students and (ii) the existence of a single allocation which is Pareto optimal from the point of view of colleges (when restricted to the set of stable matchings).

The concept of implementation is well-known in the literature. Next, we formalize it for SPE. Let $\mathcal{E}_{k}$ be the set of strategies for agent $k$ and let $\mathcal{E}=\times_{x \in C \cup S} \mathcal{E}_{x}$ be the set of strategy profiles. Associated with each strategy profile $\tilde{e} \in \mathcal{E}$, we can define a message profile $\eta(\tilde{\mathrm{e}})$, or simply $\eta$. This profile $\eta$ describes the action taken by each individual when the agents choose such strategies. A matching mechanism is described by the set of strategies allowed to each agent, and an outcome function $\gamma$ that assigns a matching to each profile of messages. We say that a matching mechanism implements a solution, say $\chi$, in Subgame Perfect Nash Equilibria if (i) for each ẽ, Subgame Perfect Equilibrium allocation for $\Gamma:=\{C$, $S ; P ; \gamma\}, \gamma(\eta(\tilde{\mathrm{e}}))$ belongs to $\chi(P)$ and (ii) for each $\mu$ in $\chi(P)$ there exists a SPE for $\Gamma$, say $\tilde{\mathrm{e}}^{\prime}$, such that $\gamma\left(\eta\left(\tilde{\mathrm{e}}^{\prime}\right)\right)=\mu$.

\section{The "students-sequentially-propose-and-colleges-choose" mechanism}

We now introduce the mechanism. But first, we fix the order in which the students play. Without loss of generality, let us assume that $s_{1}$ is the first to play, $s_{2}$ is the second and so on. Let us define the following $m+1$ stages game form. At stage $t, t=1, \ldots, m$, student $s_{t}$ selects at most one college. Thus, each student's message space coincides with the set of colleges and her option of being unmatched. At stage $m+1$, the last stage, colleges simultaneously select the set of students they want to admit, one set of students for each college. Thus, the message space of each college is $2^{S}$. Finally, the outcome function, denoted by $\Phi^{S S C}$, selects the matching defined as follows:

$\Phi^{S S C}(\eta)=\mu_{\eta}$, where for each $s_{j}$ in $S$,

$$
\mu_{\eta}\left(s_{j}\right)= \begin{cases}\eta\left(s_{j}\right) & \text { if } s_{j} \in \eta\left(\eta\left(s_{j}\right)\right) \\ s_{j} & \text { otherwise }\end{cases}
$$

\footnotetext{
${ }^{2}$ Proposition 6.4 in Roth and Sotomayor (1990) establishes that stability and pair-wise stability are equivalent concepts in college admissions problems with substitutive preferences.
} 
and, for each $c_{i}$ in $C$,

$$
\mu_{\eta}\left(c_{i}\right)=\left\{s_{j} \in \eta\left(c_{i}\right) \mid c_{i}=\eta\left(s_{j}\right)\right\}
$$

where $\eta$ is a list containing a full description of agents' messages.

Theorem 3.1 shows that our mechanism yields stable outcomes. Moreover, only one stable outcome can be reached by a Subgame Perfect Equilibrium of this mechanism. This is the student-optimal stable matching.

Theorem 3.1. Let ẽ be a SPE for $\Gamma^{S S C}:=\left\{C, S ; P ; \Phi^{S S C}\right\}$, and $\tilde{\eta}$ be the vector of messages that agents state in $\tilde{e}$. Then $\Phi^{S S C}(\tilde{\eta})=\mu^{S}(P)$, the student-optimal stable matching.

Proof. The proof is constructive. First, we present some properties that agents' messages should satisfy at equilibrium. Then, we argue that all the SPE induce the same matching, namely the student-optimal stable matching.

In order to characterize the set of SPE, we apply backward induction. At stage $m+1$, given students' messages, each college $c_{i}$ has a best response, namely, $\eta^{*}\left(c_{i}\right)=\arg \max P\left(c_{i}\right)$ on $\left\{s_{j} \mid \eta\left(s_{j}\right)=c_{i}\right\}$. ${ }^{3}$ At stage $m$, given messages for students other than $s_{m},\left(\eta\left(s_{1}\right), \ldots, \eta\left(s_{m} 1\right)\right)$, and knowing the colleges' behavior, this agent's best reply is ${ }^{4}$

$$
\eta^{*}\left(s_{m}\right)=\arg \max P\left(s_{m}\right) \text { on } \quad\left\{c_{i} \mid s_{m} \in C h_{i}\left(\left\{s_{j} \in S \backslash\left\{s_{m}\right\} \mid \eta\left(s_{j}\right)=c_{i}\right\} \cup\left\{s_{m}\right\}\right)\right\} .
$$

Notice that such a message coincides with $\mu^{S}\left(s_{m} ; P^{m}{ }^{1}\right)$, student $s_{m}$ 's mate at the student-optimal stable matching for problem $P^{m}{ }^{1}$, where $P^{m}{ }^{1}\left(c_{i}\right)=P\left(c_{i}\right)$, for each college $c_{i}$ in $C, P^{m}{ }^{1}\left(s_{m}\right)=P\left(s_{m}\right)$, and $P^{m}{ }^{1}\left(s_{j}\right)=\eta\left(s_{j}\right)$ for each student $s_{j}$ in $S \backslash\left\{s_{m}\right\}^{5}$

In order to apply an inductive argument, let us assume that $s_{k}$ 's message, $\eta^{*}\left(s_{k}\right)$ coincides with $s_{k}$ 's mate at the student-optimal stable matching for problem $P^{k}{ }^{1}$, where $P^{k}{ }^{1}\left(c_{i}\right)=P\left(c_{i}\right)$, for each college $c_{i}$ in $C, P^{k}{ }^{1}\left(s_{h}\right)=P\left(s_{h}\right)$ for each $h \geq k$, and $P^{k}{ }^{1}\left(s_{h}\right)=\eta\left(s_{h}\right)$ for each $h<k$. Note that $\mu^{S}\left(s_{k} ; P^{k}{ }^{1}\right)$ coincides with $\mu^{S}\left(s_{k} ; \hat{P}^{k}{ }^{1}\right)$ for any profile $\hat{P}^{k}{ }^{1}$ where $\hat{P}^{k}{ }^{1}\left(c_{i}\right)=P^{k}{ }^{1}\left(c_{i}\right)$ for each college, and $\hat{P}^{k}{ }^{1}\left(s_{j}\right) \in\left\{P^{k}{ }^{1}\left(s_{j}\right), P\left(s_{j}\right)\right\}$ for each student $s_{j} \in S$. Therefore, the message of student $s_{k} 1_{\text {should }}$ coincide with $\mu^{S}\left(s_{k} 1 ; P^{k}{ }^{2}\right)$, where $P^{k}{ }^{2}\left(c_{i}\right)=P\left(c_{i}\right)$ for each college $c_{i} \in C, P^{k}{ }^{2}\left(s_{h}\right)=\eta\left(s_{h}\right)$ for each $h<k \quad 1$, and $P^{k}{ }^{2}\left(s_{h}\right)=P\left(s_{h}\right)$ for each $h \geq k \quad 1$.

Finally, given that $s_{1}$ expects that the messages from each student $s_{j}$ in $S \backslash\left\{s_{1}\right\}$ should be $\eta^{*}\left(s_{j}\right)=\mu^{S}\left(s_{j}\right.$; $P)$, she would select the best college she can, provided the actions she expected from the other agents. Since $\mu^{S}(P)$ is the only stable matching which is Pareto optimal from the students' point of view, and taking into account her colleagues' best responses, her decision must be to select her mate in the studentoptimal stable matching, namely $\mu^{S}\left(s_{1} ; P\right)$.

\footnotetext{
${ }^{3}$ Notice that such a message is not the only best response. In fact, any best response for college $c_{i}$ can be expressed by the union of such a set with any set of students $S^{\prime}$ such that $c_{i} \neq \eta\left(s^{\prime}\right)$ for all $s^{\prime}$ in $S^{\prime}$. Nevertheless, all these messages are strategically equivalent. Since we are interested in equilibrium payoffs rather than equilibrium strategies, we do not pay attention to these messages.

${ }^{4}$ Note that, when $\eta^{*}\left(s_{m}\right) s_{m}$, such a strategy is equivalent to declaring any college $c_{i}$ such that $c_{i} P\left(s_{m}\right) s_{m}$. Nevertheless, since this college will not select that student, both messages are strategically equivalent. In order to provide a simple proof of our result, we assume that, whenever $\eta *\left(s_{m}\right) s_{m}$, this student's message will be her "stay unmatched" option.

${ }^{5}$ For simplicity, we identify student $s_{j}$ 's preference with college $c_{i}$, i.e. $P\left(s_{j}\right) \quad c_{i}$, whenever this college is the only one for which $c_{i} P\left(s_{j}\right) s_{j}$ holds.
} 
Consequently, at equilibrium, each student's message coincides with her mate in the student-optimal stable matching.

\section{Final remarks}

This paper introduces a mechanism that implements the student-optimal stable matching. Notice that Kara and Sönmez (1997) show that no selection of the core can be implemented in Nash Equilibrium.

In the many-to-one matching problem, Alcalde and Romero-Medina (2000) show that, when students play simultaneously, the role played by colleges and students can be exchanged without affecting the results. They implement the core correspondence, no matter whether colleges or students make the offers (i.e. play at the first stage). Nevertheless, a similar result cannot be established if offers are made sequentially. We conclude by providing an example that shows the above-mentioned asymmetry.

Example 4.1. Let $\{C, S ; P\}$ be a three colleges-four students problem. The following table summarizes agents' preferences.

$$
\begin{aligned}
& P\left(s_{1}\right)=c_{3} c_{2} c_{1} \\
& P\left(s_{2}\right)=c_{2} c_{1} c_{3} \\
& P\left(s_{3}\right)=c_{2} c_{1} c_{3} \\
& P\left(s_{4}\right)=c_{3} c_{1} c_{2} \\
& P\left(c_{1}\right)=\left(s_{3} s_{4}\right)\left(s_{2} s_{4}\right)\left(s_{2} s_{3}\right)\left(s_{1} s_{4}\right)\left(s_{1} s_{3}\right)\left(s_{1} s_{2}\right) s_{4} s_{3} s_{2} s_{1} \\
& P\left(c_{2}\right)=s_{4} s_{3} s_{2} s_{1} \\
& P\left(c_{3}\right)=s_{3} s_{4} s_{1} s_{2}
\end{aligned}
$$

Let us consider the "colleges-sequentially-propose-and-students-choose" mechanism, $\Gamma^{C s S}$. This is a symmetrical version of $\Gamma^{S S C}$ in which colleges propose in a sequential way. We see that two interesting features which are satisfied by the family of mechanisms $\Gamma^{S S C}$ are not satisfied by a mechanism in $\Gamma^{C S S}$. First, some SPE outcomes can be unstable, relative to agents' preferences. In order to show this, let us suppose that the order in which colleges sequentially propose is $c_{1}, c_{2}$ and $c_{3}$. There is a SPE with messages $\tilde{\eta}\left(c_{1}\right)=\left(s_{1} s_{2}\right), \quad \tilde{\eta}\left(c_{2}\right)=\left(s_{4}\right), \quad \tilde{\eta}\left(c_{3}\right)=\left(s_{3}\right), \quad \tilde{\eta}\left(s_{1}\right)=\left(c_{1}\right), \quad \tilde{\eta}\left(s_{2}\right)=\left(c_{1}\right)$, $\tilde{\eta}\left(s_{3}\right)=\left(c_{3}\right)$ and $\tilde{\eta}\left(s_{4}\right)=\left(c_{2}\right)$. Notice that $\Gamma^{C s S}(\tilde{\eta})$ is unstable because the pair $\left\{c_{1} s_{3}\right\}$ blocks it. Secondly, the SPE outcomes set depends on the order in which colleges play. Indeed, let us consider the order for colleges in which $c_{2}$ proposes first, then $c_{3}$, and finally $c_{1}$ is the last to propose. In such a case, the only SPE outcome is $\mu\left(c_{1}\right)=\left(s_{1} s_{2}\right), \mu\left(c_{2}\right)=\left(s_{3}\right)$ and $\mu\left(c_{3}\right)=\left(s_{4}\right)$, which is different from the SPE outcome when college $c_{1}$ is the first to play. 


\section{Acknowledgements}

We wish to thank Salvador Barberà and Luis C. Corchón for their comments. Alcalde's work is partially supported by the Institut Valencià d'Investigacions Econòmiques and the Spanish Ministerio de Educación y Cultura under project BEC 2001-0535. Romero-Medina acknowledges financial support from the Spanish Ministerio de Educación y Cultura under project BCE-2002-02194 and Comunidad de Madrid under project 02303.

\section{References}

Alcalde, J., Romero Medina, A., 2000. Simple mechanisms to implement the core of college admissions problems. Games and Economic Behavior 31, 294302.

Alcalde, J., Pérez Castrillo, D., Romero Medina, A., 1998. Hiring procedures to implement stable allocations. Journal of Economic Theory 88, 469480.

Kara, T., Sönmez, T., 1997. Implementation of college admissions rules. Economic Theory 9, 197218.

Roth, A.E., Sotomayor, M., 1990. Two Sided Matching: A Study in Game Theoretic Modeling and Analysis. Cambridge University Press, New York. 